\title{
Charge bag model of superconductivity in intercalated layered compounds
}

\author{
S N BEHERA and D M GAITONDE \\ Institute of Physics, Bhubaneswar 751005 , India
}

\begin{abstract}
In certain layered compounds the superconducting transition temperature $\left(T_{c}\right)$ is enhanced on intercalation. The superconductivity in these materials arises over a charge density wave background. To explain this enhancement in $T_{c}$ we propose the charge bag model analogous to the spin bag model proposed earlier by Schrieffer as a mechanism for high $T_{c}$ superconductivity.
\end{abstract}

Keywords. Layered compounds; charge density wave; intercalation.

\section{Introduction}

The transition metal dichalcogenides being layered compounds (Wilson et al 1975) are quasi-two-dimensional systems. The low dimensionality leads to an inherent Fermi surface instability which brings about a charge density wave (CDW) transition. However if the nesting of the Fermi surface is not perfect then this transition takes place only in the directions of nesting. As a result, the transition will lead to an anisotropic semiconducting state with a pseudo-gap in the electronic energy spectrum. Therefore there exists the possibility for some of these systems to undergo the superconducting (SC) transition on cooling. The highest $T_{c}$ of $7 \cdot 2 \mathrm{~K}$ is found in $2 \mathrm{H}-\mathrm{NbSe}_{2}$ whose Peierls transition temperature $T_{p}=33 \mathrm{~K}$. On the other hand in $2 \mathrm{H}-\mathrm{TaSe}_{2}$, $T_{p}=120 \mathrm{~K}$ and $T_{c}=0.2 \mathrm{~K}$. The higher the $T_{p}$, the larger is the area of the nested Fermi surface, and therefore the lower is $T_{c}$. On intercalating with organic molecules, the layer spacing increases (Ginzburg and Krizhnits 1982) making these systems more two-dimensional. This will result in better nesting, which is expected to raise $T_{p}$ and suppress $T_{c}$. Contrary to this expectation $T_{c}$ rises from $0.2 \mathrm{~K}$ to $4.5 \mathrm{~K}$ in the case of $2 \mathrm{H}-\mathrm{TaSe}_{2}$ and from $0.8 \mathrm{~K}$ to $2 \mathrm{~K}$ in the case of $2 \mathrm{H}-\mathrm{TaS}_{2}$, the values of $T_{p}$ being $120 \mathrm{~K}$ and $80 \mathrm{~K}$ for the above two systems respectively. This suggests that the better the nesting of the Fermi surface, the larger is the increase in $T_{c}$ on intercalation. Thus the increase in $T_{c}$ cannot be understood as arising from the suppression of the CDW state.

To explain the increase in $T_{c}$ on intercalation, we propose the charge bag mechanism analogous to the spin bag mechanism (Schrieffer et al 1988) of high $T_{c}$ superconductivity. We assume that the intercalant organic molecules act as donors and inject charge carriers into an essentially rigid CDW conduction band of the transition metal dichalcogenide layers. These carriers interact by the exchange of the quanta of the collective modes of the CDW state which gives rise to an effective attractive interaction. This results in pairing and hence SC. In $\$ 2$ the collective modes of the $\mathrm{CDW}$ are calculated and the effective interaction between injected electrons is determined. In $\$ 3$ a mean field theory of SC is considered and the reasons for the enhancement of $T_{c}$ are discussed. 


\section{Collective modes of the CDW and effective interaction between injected carriers}

A mean field theory for the CDW state can be developed starting from an electronphonon hamiltonian. We assume a half-filled tight-binding band which ensures a perfectly nested Fermi surface with $Q=(\pi / a, \pi / a)$. Performing a canonical transformation, the effective electronic Hamiltonian to $o\left(g^{2}\right)$ (where $g$ is the electron-phonon coupling constant) is given by

$$
H=\sum_{k, \alpha} \varepsilon_{k} c_{k, \alpha}^{\dagger} c_{k, \alpha}-\frac{V}{2} \sum_{k, k^{\prime}, q, \alpha, \alpha^{\prime}} c_{k+q, \alpha}^{\dagger} c_{k, \alpha} c_{k^{\prime}-q, \alpha^{\prime}}^{\dagger} c_{k^{\prime}, \alpha^{\prime}}
$$

The mean field Hamiltonian for the CDW state is given by

$$
H_{\mathrm{MF}}=\sum_{k, \alpha}^{F B Z} \varepsilon_{k} c_{k, \alpha}^{\dagger} c_{k, \alpha}+W \sum_{k, \alpha}^{R B Z}\left[c_{k+Q, \alpha}^{\dagger} c_{k, \alpha}+\text { h.c. }\right] \text {. }
$$

Here $\varepsilon_{k}=-2 t\left[\cos k_{x} a+\cos k_{y} a\right], W=-V\left\langle\rho_{Q}\right\rangle=g\left\langle b_{Q}+b_{-Q}^{\dagger}\right\rangle$ is the CDW order parameter, $\rho_{Q}$ is the $Q$ th Fourier component of the charge density and $b_{q}^{\dagger}$ is a phonon creation operator. This hamiltonian can be diagonalized by the Bogoliubov transformation

$$
c_{k, \alpha}=\cos \phi_{k} \gamma_{k, \alpha}^{c}+\sin \phi_{k} \gamma_{k, \alpha}^{v}, c_{k+Q, \alpha}=-\sin \phi_{k} \gamma_{k, \alpha}^{c}+\cos \phi_{k} \gamma_{k, \alpha}^{v},
$$

which reduces equation (2) to

$$
H_{\mathrm{MF}}=\sum_{k, \alpha} E_{k}\left[\gamma_{k, \alpha}^{c \dagger} \gamma_{k, \alpha}^{c}-\gamma_{k, \alpha}^{v \dagger} \gamma_{k, \alpha}^{v}\right]
$$

where $E_{k}=\left(\varepsilon_{k}^{2}+W^{2}\right)^{1 / 2}$ is the quasi-particle energy, $\cos 2 \phi_{k}=\varepsilon_{4} / E_{k}$ and $\sin 2 \phi_{k}=$ $-W / E_{k}$. The superscripts $c(v)$ denote conduction (valence) band operators. The CDW gap is given by

$$
1=V \sum_{k, \alpha} \frac{\tanh \left(\beta E_{k} / 2\right)}{2 E_{k}}
$$

The diagonalized CDW Hamiltonian corresponds to the 'pure' compounds.

On intercalation, electrons are added to the conduction band. These 'doped' electrons can be described by the operators $\gamma_{k, \alpha}^{c}$ and a chemical potential $\mu$. The calculation of the collective modes is carried out using the method of Kulik et al (1981), which can be formulated by introducing the Nambu operators

$$
\hat{\psi}_{k, \alpha}^{\dagger}=\left(c_{k, \alpha}^{\dagger}, c_{k+Q . \alpha}^{\dagger}\right) \text {. }
$$

Within the RPA, the collective mode frequencies are determined from

$$
\frac{2}{V}+\chi^{i i}(q, \omega)=0 \quad i=1,2,
$$

where the susceptibility functions

$$
\chi^{i i}=\sum_{k, k^{\prime}, \alpha, \alpha^{\prime}}\left\langle\left\langle\hat{\psi}_{k, \alpha}^{\dagger}(t) \hat{\sigma}_{i} \hat{\psi}_{k+q, \alpha}(t) ; \hat{\psi}_{k^{\prime}+q, \alpha^{\prime}}^{\dagger}(0) \hat{\sigma}_{i} \hat{\psi}_{k^{\prime}, \alpha^{\prime}}(0)\right\rangle\right\rangle_{\omega}
$$

are evaluated using $H_{\mathrm{MF}}$. For $q=0$ we find the frequency of the amplitude mode 
(AM) from (7) using $\chi^{11}$ and it turns out to be $\omega_{+}=2 W$ while $\chi^{22}$ gives the phase mode (PM) frequency as $\omega_{-}(q)=\sqrt{ } 2$ taq in the long wavelength limit.

So far our formulation has been purely in terms of the electronic charge densities. However the CDW state can equivalently be viewed as a phonon condensate. In this case the fluctuation quanta correspond to phonons of the system after it has undergone a periodic lattice distortion. Due to the superlattice formation there will be an acoustic branch (PM) and a zone-folded optic branch (AM). Thus the condensed electronphonon system can be described by $H^{\prime}=H_{\mathrm{MF}}+H_{\mathrm{ph}}^{\prime}+H_{e-p}^{\prime}$ where

$$
H_{\mathrm{ph}}^{\prime}=\sum_{i=1}^{2} \sum_{q} \omega_{q}^{i} d_{q}^{i \dagger} d_{q}^{i}
$$

and

$$
H_{e-p}^{\prime}=g^{\prime} \sum_{i=1}^{2} \sum_{k, q, \alpha} \hat{\psi}_{k+q, \alpha}^{\dagger} \hat{\sigma}_{i} \hat{\psi}_{k, \alpha}\left(d_{q}^{i}+d_{-q}^{i \dagger}\right) .
$$

Here $d_{q}^{1 \dagger}$ creates an AM phonon and $d_{q}^{2 \dagger}$ creates a PM phonon, $g^{\prime}$ being the renormalized electron-phonon coupling constant for the condensed phase. The renormalized phonon frequencies are taken to be the same as the RPA results. The effective interaction (to $o\left(g^{\prime 2}\right)$ ) between conduction band electrons due to the exchange of the collective modes is found to be

$$
H_{\mathrm{eff}}=g^{\prime 2} \sum_{k, k^{\prime}, q, \alpha, \alpha^{\prime}}\left[f_{1}\left(k, k^{\prime}, q\right)+f_{2}\left(k, k^{\prime}, q\right)\right] \gamma_{k+q, \alpha^{\prime}}^{c \dagger} \gamma_{k^{\prime}, \alpha^{\prime}}^{c \dagger} \gamma_{k^{\prime}+q, \alpha^{\prime}}^{c} \gamma_{k, \alpha}^{c}
$$

where

$$
\begin{aligned}
f_{1}\left(k, k^{\prime}, q\right) & =\frac{\omega_{q}^{A M} \sin \left(\phi_{k}+\phi_{k+q}\right) \sin \left(\phi_{k^{\prime}}+\phi_{k^{\prime}+q}\right)}{\left(E_{k^{\prime}+q}-E_{k^{\prime}}\right)^{2}-\omega_{q}^{A M 2}}, \\
f_{2}\left(k, k^{\prime}, q\right) & =\frac{\omega_{q}^{P M} \sin \left(\phi_{k}-\phi_{k+q}\right) \sin \left(\phi_{k^{\prime}}-\phi_{k^{\prime}+q}\right)}{\left(E_{k^{\prime}+q}-E_{k^{\prime}}\right)^{2}-\omega_{q}^{P M 2}} .
\end{aligned}
$$

For small momentum transfer, $\phi_{k} \approx \phi_{k+q}$. Thus the PM channel can be neglected while the AM channel gives rise to an attractive interaction.

\section{SC in the CDW conduction band}

The attractive interaction mediated by $\mathrm{AM}$ exchange can give rise to pairing and hence to SC. For simplicity we assume the strength of the attractive interaction to be a constant $(-\lambda)$. Thus the BCS-like hamiltonian for the conduction band electrons becomes

$$
H_{\mathrm{SC}}=\sum_{k, \alpha}\left(E_{k}-\mu\right) \gamma_{k, \alpha}^{c \dagger} \gamma_{k, \alpha}^{c}-\lambda \sum_{k, k^{\prime}} \gamma_{k, \uparrow}^{c \dagger} \gamma_{-k, \downarrow}^{c^{\dagger}} \gamma_{-k^{\prime}, \downarrow}^{c} \gamma_{k^{\prime}, \uparrow}^{c}
$$

Within the mean field approximation we get the usual gap equation

$$
\frac{2}{\lambda}=\sum_{k} \frac{\tanh \left(\beta e_{k} / 2\right)}{e_{k}},
$$

where $e_{k}=\left[\left(E_{k}-\mu\right)^{2}+\Delta^{2}\right]^{1 / 2}$ and $\Delta$ is the SC gap. This equation has to be 
supplemented by another equation for the chemical potential given by

$$
N_{0}=2 \sum_{k} f\left(E_{k}-\mu\right)
$$

where $N_{0}$ is the number of carriers injected into the conduction band and $f$ is the Fermi function. The SC transition temperature is given by

$$
k_{B} T_{c}=1 \cdot 13\left(\omega_{+}\right) \exp \left(-\frac{1}{N(\mu) \lambda}\right) .
$$

Here $N(\mu)$ is the quasi-particle density-of-states (DOS) in the CDW state and the BCS cut-off energy now becomes $\omega_{+}$, the AM frequency. $N(\mu)$ is given by

$$
N(\mu)=[\rho(0) \mu] /\left(\mu^{2}-W^{2}\right)^{1 / 2},
$$

where $\rho(0)$ is the DOS at the Fermi surface of the original tight-binding band. Thus for low carrier concentration $N(\mu)$ is expected to be large. Also the cut-off energy $\omega_{+}=2 W$ increases with increasing values of $T_{p}$. Thus a satisfactory explanation is found for the enhancement of $T_{c}$ which is in qualitative agreement with experimental data.

\section{References}

Ginzburg V L and Krizhnits D A (eds) 1982 in High temperature superconductivity, (New York: Consultant Bureau) p. 111

Kulik I O et al 1981 J. Low Temp. Phys. 43591

Schrieffer J R et al 1988a Phys. Rev. Lett. 60944

Schrieffer J R et al 1988b Phys. Rev. B39 11663

Wilson J A et al 1975 Adv. Phys. 24117 